The Astrophysical Journal, $191:$ L121-L125, 1974 August 1

(C) 1974. The American Astronomical Society. All rights reserved. Printed in U.S.A.

\title{
A NEW INFRARED COMPLEX AND MOLECULAR CLOUD IN ORION
}

\author{
I. Gatley, ${ }^{*}$ E. E. Becklin, $†$ K. Matthews, ${ }^{*}$ G. Neugebauer, $†$ M. V. Penston $\ddagger$ and N. Scoville $\S$ \\ Received 1974 A pril 30
}

\section{ABSTRACT}

A new complex of infrared sources $\sim 1^{\prime}$ across has been discovered in Orion. Associated with this cluster is an extended $\sim 6^{\prime}$ molecular cloud producing intense CO emission. Its measured infrared luminosity is less than $\sim 500 L_{\odot}$. The complex is hypothesized to be composed of pre-main-sequence sources. Several arguments suggest that these stars heat dust in the larger molecular cloud and thus cause the strong CO emission. The complex is not associated with a known visible or radio continuum feature, and searches over a $0.7 \times 0.7$ field show no other comparable unknown $2.2-\mu$ sources.

Subject headings: infrared sources - Orion Nebula

\section{INTRODUCTION}

In the infrared, the Orion Nebula has been characterized by emission in two main regions. One-the NeyAllen source-is centered on the Trapezium stars while the second region is $\sim 1^{\prime}$ northwest of this. In the second region an unresolved source-generally called the Becklin-Neugebauer source-dominates the emission at wavelengths less than $\sim 10 \mu$. An extended source-the Kleinmann-Low Nebula-dominates the emission at longer wavelengths (see, e.g., WynnWilliams and Becklin 1974) and has centered on it a CO molecular cloud (Liszt et al. 1974). We will denote this second region of infrared and molecular emission as the "KL Nebula." In this Letter another complex of infrared and molecular emission which is $\sim 12^{\prime}$ northeast of the Trapezium is discussed. We have labeled this newly found complex as the Orion molecular cloud 2 (OMC 2). It was discovered during infrared measurements of stars in a program unrelated to the present letter.

\section{OBSERVATIONS}

The infrared data were obtained during the period 1973 December through 1974 March at the 24-, 60-, and 100-inch $(61-\mathrm{cm}),(1.5-\mathrm{m})$, and $(2.5-\mathrm{m})$ telescopes on Mount Wilson and the 200-inch (5-m) Hale telescope in a conventional manner. Measurements were made with focal plane diaphragms from $5^{\prime \prime}$ to $1^{\prime}$ in diameter in photometric bands centered at 1.6, 2.2, 3.5, 4.8, 8.7, $9.5,10.1,11.2,12.7$, and $20 \mu$. The widths and calibration of the majority of these bands have been listed by Wilson et al. (1972); bands at $8.7 \mu(\Delta \lambda=1.2 \mu), 9.5 \mu$ $(\Delta \lambda=1.5 \mu), 11.2 \mu(\Delta \lambda=1.5 \mu)$, and $12.7 \mu(\Delta \lambda=$ $1.3 \mu)$ were utilized to look for the $10-\mu$ "silicate" absorption feature.

The $\mathrm{CO}(J=1 \rightarrow 0)$ and $\operatorname{CS}(J=3 \rightarrow 2)$ data were obtained in 1974 February with the 16-foot $(6.3-\mathrm{m})$

* California Institute of Technology, 1201 East California Blvd., Pasadena, CA 91109.

$\dagger$ Hale Observatories, California Institute of Technology, Carnegie Institution of Washington.

$\ddagger$ Royal Greenwich Observatory, Herstmonceux Castle, Hailsham, Sussex BN27 1RP, England.

$\S$ Owens Valley Radio Observatory, California Institute of Technology. antenna of the Millimeter-Wave Observatory, ${ }^{1}$ a frontend receiver developed at Bell Telephone Laboratories, and a 40 -channel filter bank $\left(0.65 \mathrm{~km} \mathrm{~s}^{-1}\right.$ per channel). The half-power beamwidth was $2^{\prime}$. All observed antenna temperatures were corrected for atmospheric attenuation.

\section{RESULTS}

The location of OMC 2 relative to the Trapezium is presented in figure 1. In figure 2 it is shown that at $2.2 \mu$, OMC 2 contains five separate components within a region whose dimensions are about $1^{\prime}$; some are resolved and others unresolved with a 7".5 resolution. No counterpart to the infrared sources is present on a plate with an effective wavelength of $7200 \AA$ taken by D. Osterbrock at the Newtonian focus of the Mount Wilson Hooker telescope. From the infrared energy distributions presented in figure 3 , it is seen that the infrared color temperature of the brightest infrared component, OMC $2 /$ IRS 3 , is in the range of $500^{\circ} \mathrm{K}$. The 1950.0 coordinates of this source are: right ascension $=5^{\mathrm{h}} 32^{\mathrm{m}} 59^{\mathrm{s}} .1 \pm$ $0 \mathrm{~s}$, declination $=-5^{\circ} 12^{\prime} 10^{\prime \prime} \pm 1^{\prime \prime}$.

The CO emission shown in figure 1 peaks within $1^{\prime}$ of the infrared complex, but extends outwards about $6^{\prime}$. The maximum CO antenna temperature of $49^{\circ} \mathrm{K}$ $\left(V_{\mathrm{LSR}}=10.9 \mathrm{~km} \mathrm{~s}^{-1}, \Delta V=2.9 \mathrm{~km} \mathrm{~s}^{-1}\right)$ implies a $\mathrm{CO}$ brightness and excitation temperature of $53^{\circ} \mathrm{K}$. The intensity of the $\mathrm{CO}$ emission from this region is therefore comparable to that of the $\mathrm{KL}$ Nebula $\left(T_{B}=75^{\circ} \mathrm{K}\right.$, Liszt et al. 1974). The observations in the CS lines show a corrected antenna temperature of $3.3^{\circ} \mathrm{K}$ at the position of OMC 2 , while only $1^{\prime}$ west and $2^{\prime}$ south the temperature has dropped to about $1^{\circ} \mathrm{K}$. Between the same positions the $\mathrm{CO}$ intensity changes less than 10 percent.

The CO emission shows a pronounced north-south orientation, and clearly extends beyond the southern extent of the present observations. In the sense that a decrease exists in the line velocity and an increase in the line width in the direction of the $\mathrm{KL}$ Nebula $\left(V_{\mathrm{LSR}}=\right.$

${ }^{1}$ The Millimeter-Wave Observatory is operated by Electrical Engineering Research Laboratory, the University of 'Texas at Austin, with support from the National Aeronautics and Space Administration, the National Science Foundation, and McDonald Observatory. 
$9 \mathrm{~km} \mathrm{~s}^{-1}, \Delta V=6.5 \mathrm{~km} \mathrm{~s}^{-1}$ ) there may be a smooth transition of the enveloping gas between the two regions. It is impossible from these observations to tell if the $\mathrm{CO}$ flux has fine structure on the scale observed in the infrared sources or if there is an extremely low surface brightness infrared component the size of the extended structure evident in the $\mathrm{CO}$ emission.

In addition to the map shown in figure 2, searches were made over extended regions of the Orion Nebula. An area $\sim 40^{\prime} \times 40^{\prime}$ centered on the Trapezium was scanned at $2.2 \mu$ with a $1^{\prime}$ aperture to a flux level of $1 \mathrm{fu}\left(1 \mathrm{fu}=10^{-26} \mathrm{~W} \mathrm{~m} \mathrm{~m}^{-2} \mathrm{~Hz}^{-1}\right)$, about one-fifth that observed from OMC 2. Of the sources found, all but the KL Nebula and OMC 2 were identified with visible stars (Penston 1973). A 2.2- $\mu$ survey to a flux limit of 0.1 fu and covering an area $6^{\prime} \times 6^{\prime}$ centered on OMC 2 showed only the sources associated with OMC 2 and visibly identified stars. A search at $20 \mu$ of the area covered in figure 2 indicates there are no $20-\mu$ sources stronger than $200 \mathrm{fu}$ in a $20^{\prime \prime}$ beam. A less complete, but also negative, $\mathrm{CO}$ survey was carried out over the region of $\sim 1^{\circ} \times 1^{\circ}$ centered on the KL Nebula.

In summary, observations now show two molecular condensations in the Orion Nebula region. Each contains a multiplicity of infrared sources and is of comparable size, but neither is associated with visible features. The $\mathrm{CO}$ peak brightness temperatures of the two are similar, while the line widths of the $\mathrm{CO}$ in the $\mathrm{KL}$ Nebula are twice those in OMC 2. In the infrared, OMC 2 is fainter than the $\mathrm{KL}$ Nebula in its integrated $2.2-\mu$ flux by a factor of 10 and in its $20-\mu$ flux by a factor of 100 . In contrast, the energy distribution of OMC 2/IRS 3 is like that of the Becklin-Neugebauer source except that the latter exhibits a strong silicate absorption feature and has a much steeper slope between 2.2 and $1.6 \mu$. Furthermore, these two sources are less than $5^{\prime \prime}$ in diameter at $2.2,10$, and $20 \mu$. A resemblance also exists between the spectrum of the extended source OMC 2/IRS 4 and that of the extended KL Nebula at wavelengths longer than $5 \mu$, although its luminosity is lower by a factor close to 1000 .

\section{DISCUSSION}

The nature of the infrared sources in OMC 2 is unknown, but they are probably not purely reddened stars. Since the luminosity integrated over wavelengths shorter than $20 \mu$ is less than $500 L_{\odot}$, the sources cannot be young, massive $\mathrm{O}$ - and B-type stars embedded in dust unless there is unexpectedly large emission at wavelengths in excess of $20 \mu$. In addition, the energy distributions are inconsistent with those of highly reddened stars with photospheric temperatures > $2000^{\circ} \mathrm{K}$. Specifically, if the color of OMC $2 / \operatorname{IRS} 3$ between 1.6 and $2.2 \mu$ is assumed to be entirely due to reddening of a Rayleigh-Jeans blackbody spectrum, the visual extinction corresponds to $\sim 30 \mathrm{mag}$. If the spectrum of OMC 2/IRS 3 is corrected for this extinction with a normal reddening law, a large excess flux is still observed at 5, 10, and $20 \mu$. Finally, OMC 2/IRS 1 and OMC 2/IRS 4 both are clearly extended and thus cannot be ordinary stars.
The arguments above cannot exclude the possibility that the radiation comes both from stars and an additional radiation component. If this obtains, it is probable that the excess arises as thermal emission from dust which is heated by a central object. Ney, Strecker, and Gehrz (1973) have observed thermal radiation at 10 and $20 \mu$ from six early-type stars in Orion. These sources have angular sizes about $25^{\prime \prime}$ and thus differ markedly from OMC $2 / \operatorname{IRS} 3$. The emission from the extended sources cannot be free-free since observations at $8 \mathrm{GHz}$ set a limit of less than $0.25 \mathrm{fu}$ from a $40^{\prime}$ area centered on OMC 2 (Gezari and Crutcher 1974); at this limit a $15^{\prime \prime}$ source is optically thin at $8 \mathrm{GHz}$.

The absence of the silicate feature around $10 \mu$ can be used to tell something about the cold dust in the vicinity of OMC 2. This feature has been observed, in particular, in discrete sources within the KL Nebula (Gillett and Forrest 1973; Rieke and Low 1973), and in the intervening material to the galactic center (Woolf 1973; Aitken and Jones 1973). If the unproven assumption is made that the visual extinction is proportional to the depth of the $10-\mu$ absorption feature, then the data indicate that the visual extinction to OMC 2 is much less than the 30 mag present towards the galactic center.

The apparently smooth extension of $\mathrm{CO}$ emission from OMC 2 toward the KL Nebula should not be taken to suggest that the gas density is constant over this large region, since the $\mathrm{CO}$ transition is undoubtedly saturated and probably thermalized. If the abundance ratio $\left[\mathrm{CS} / \mathrm{H}_{2}\right]$ is constant, the observed change in the $\mathrm{CS}$ intensity can result from a decrease in the gas density by a factor of 3 (see Scoville and Solomon 1974).

Some properties of the molecular cloud can be crudely estimated from the observed $\mathrm{CO}$ lines. From the virial theorem and the line width, the hydrogen density $\bar{n}_{\mathrm{H}_{2}} \simeq 10^{4} \mathrm{~cm}^{-3}$, the total mass is $\simeq 10^{3} M_{\odot}$, and the dynamical time scales are $\sim 5 \times 10^{5}$ years. The cooling rate for the cloud by $\mathrm{CO}$ molecules at the observed temperatures is $1 L_{\odot}$, much less than the observed infrared luminosity.

The relation between the infrared and $\mathrm{CO}$ emissions is probably casual rather than coincidental. Goldreich and Kwan (1974) show that in molecular clouds the hydrogen gas will be closely coupled to the dust in regions of $n_{\mathrm{H}_{2}}>10^{4} \mathrm{~cm}^{-3}$. In the absence of energy sources, the dust grains, the $\mathrm{H}_{2}$, and the $\mathrm{CO}$, which are almost in thermal equilibrium, will rapidly cool. Thus luminous sources which heat the dust are probably required for strong $\mathrm{CO}$ emissions. The lower temperature of the $\mathrm{CO}$ in OMC 2 as compared to the $\mathrm{KL}$ Nebula follows from the lower luminosity of the sources in the former. In addition, the $\mathrm{CO}$ temperatures will decrease only as a weak function of the distance from the source, consistent with the large extent of the $\mathrm{CO}$ cloud. We feel the good qualitative agreement of these predictions with the observations is strong evidence that in hot molecular clouds the $\mathrm{CO}$ is heated by luminous near-infrared sources within the cloud.

The evolutionary state of the infrared sources in OMC 2 is very uncertain. As has been suggested for the Becklin-Neugebauer source and several other similar 


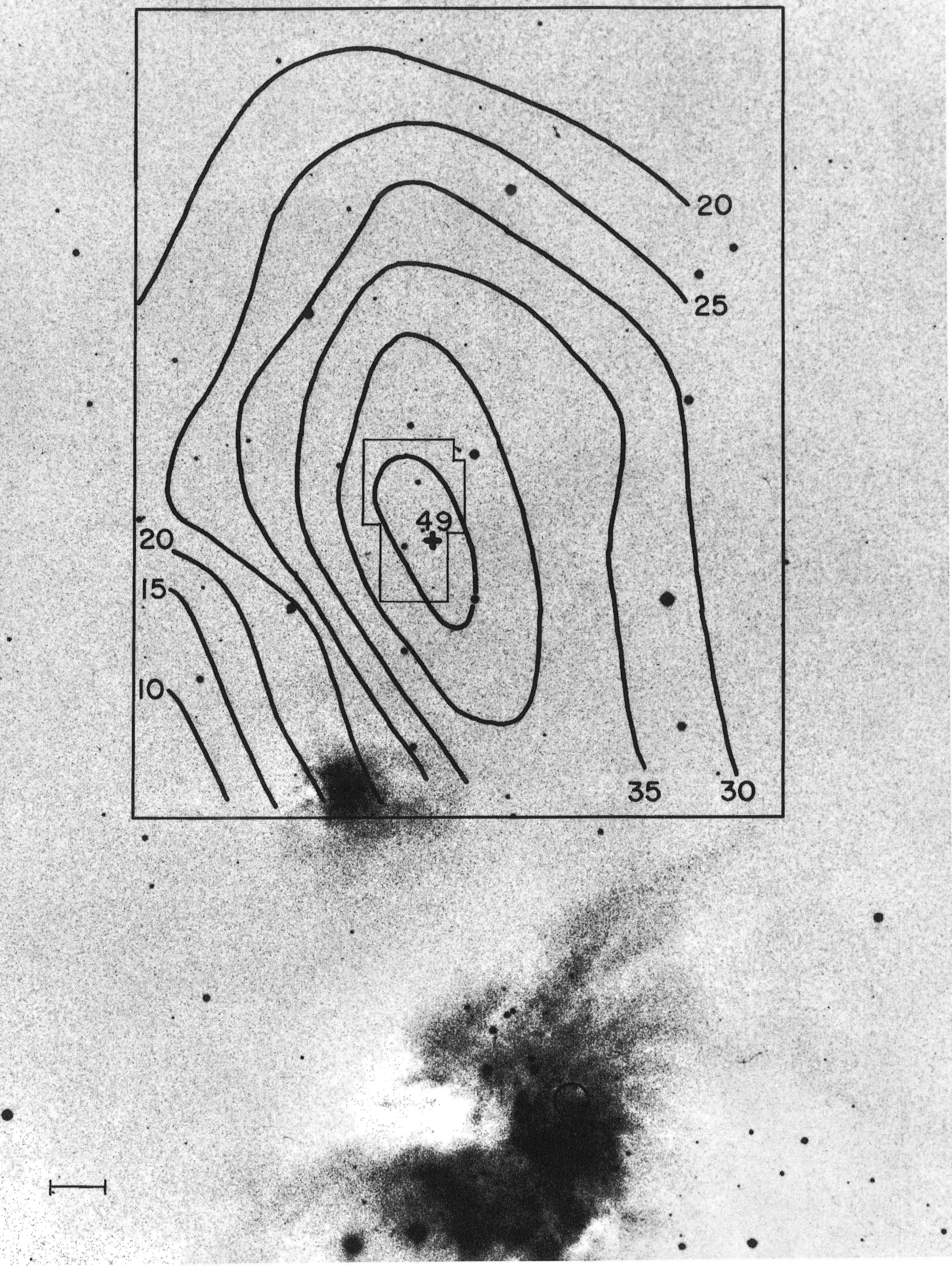

FIG. 1.-The location of OMC 2 and contours of the CO antenna temperatures are shown on a short exposure 103aJ 48-inch plate taken by R. Brucato. The circle marks the approximate position of the KL Nebula. The insert corresponds to the outline of fig. 2 . The CO velocity varies from 11.9 to $10.9 \mathrm{~km} \mathrm{~s}^{-1}$ from the top to the middle of the CO map. The beam size of the CO measurements was $2^{\prime}$ and the positional accuracy is about $1^{\prime}$. The horizontal bar at the bottom left is $1^{\prime}$ long; north is up and east is left. 


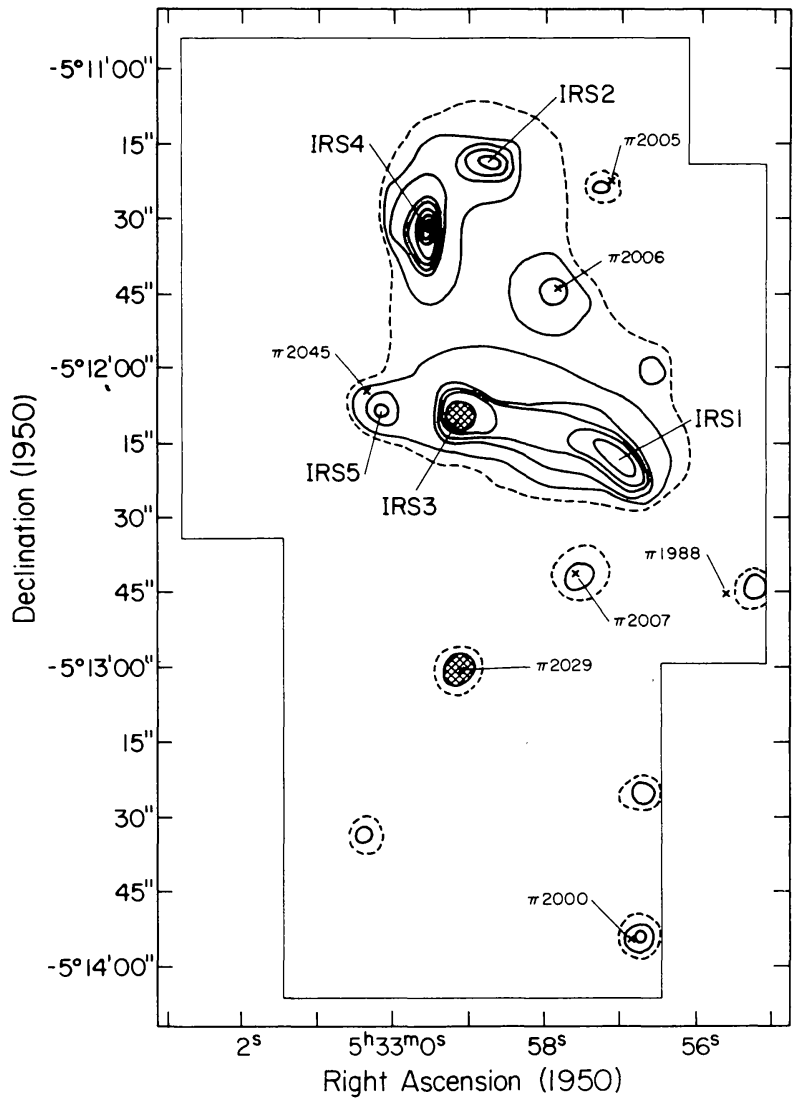

Fig. 2.-A 2.2- $\mu$ map of OMC 2 made by taking successive right ascension scans with a resolution of $7 " .5$ is shown. The difference in the contour levels is $\sim 0.02 \mathrm{fu}$ in a 7".5 beam, and the dashed curve represents a half-contour interval. The five discrete infrared sources are labeled IRS 1 through IRS 5. IRS 2 and IRS 3 are unresolved; the flux density of IRS 3 is $1.3 \mathrm{fu}$. The objects numbered with $\pi$ refer to visible stars identified by Parenago (1954).

infrared objects (see Wynn-Williams and Becklin 1974), it is possible that the objects may be protostars. Alternatively, some of the sources could be highly absorbed stars with excess long-wavelength emission lying in or near the molecular cloud. Theories of stellar evolution place the main-sequence lifetime of stars with luminosities of $\sim 100 L_{\odot}$ as $>10^{8}$ years (see, e.g., Iben 1967). It is, we think, unlikely that a complex such as

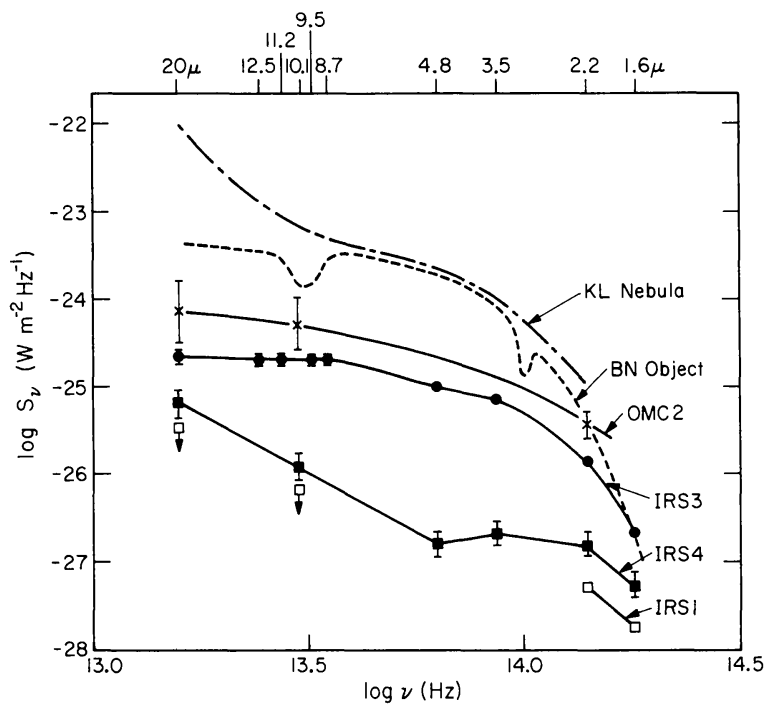

FIg. 3.-Energy distributions of the central 7".5 of OMC 2/ IRS 1, OMC/IRS 3 and OMC/IRS 4 are shown. The uncertainties include the effects of contamination in the comparison beam of the two-beam photometer. The total flux from a $1^{\prime}$ aperture taken with a $3^{\prime}$ chopper spacing is labeled OMC 2 . At 10 and $20 \mu$ only upper limits were measured directly; the lower bounds on these data points were obtained by adding together the known fluxes of the individual sources. The energy distributions of the KL Nebula through a $30^{\prime \prime}$ diameter aperture and the $\mathrm{BN}$ source are shown for comparison (Becklin, Neugebauer, and Wynn-Williams 1973; Sutton, Becklin, and Neugebauer 1974; Gillett and Forrest 1973).

observed in OMC 2 can maintain its integrity with respect to the molecular cloud over this time span. For example, if the relative velocity of the sources with respect to the condensation were $1 \mathrm{~km} \mathrm{~s}^{-1}$, then the time scale for $1^{\prime}$ movement would be only $\sim 10^{5}$ years. We thus favor the view that the infrared sources in OMC 2 must be very young objects.

We thank S. Beckwith, J. Carrasco, G. Hancock, K. Jefferts, and P. Solomon for their assistance with the observations and J. Frogel, M. Werner, and B. Zuckerman for discussions. This work was supported by National Aeronautics and Space Administration grant NGL 05-002-207 and National Science Foundation grant GP 35545X.

\section{REFERENCES}

Aitken, D. K., and Jones, B. 1973, A p. J., 184, 127

Becklin, E. E., Neugebauer, G., and Wynn-Williams, C. G. 1973, $A p . J$. (Letters), 182, L7.

Gezari, D. Y., and Crutcher, R. M. 1974, private communication. Gillett, F. C., and Forrest, W. J. 1973, A p. J., 179, 483.

Goldreich, P., and Kwan, J. 1974, A p. J., 189, 441.

Iben, I. 1967, Ann. Rev. Astr. and A p., 5, 571 .

Liszt, H. S., Wilson, R. W., Penzias, A. A., Jefferts, K. B., Wannier, P. G., and Solomon, P. M. 1974, 190, 557.

Ney, E. P., Strecker, D. W., and Gehrz, R. D. 1973, A p. J., 180, 809.

Parenago, P. P. 1954, Works of the Sternberg Astronomical Institute, Vol. 25,
Penston, M. V. 1973, Ap. J., 183, 505.

Rieke, G. H., and Low, F. J. 1973, Bull. $A A S$, 5, 436.

Scoville, N. Z., and Solomon, P. M. 1974, A p. J. (Letters), 187, L67.

Sutton, E., Becklin, E. E., and Neugebauer, G. 1974, $A p . J$. (Letters), 190, L69

Wilson, W. J., Schwartz, P. R., Neugebauer, G., Harvey, P. M., and Becklin, E. E. 1972, Ap. J., 177, 523.

Woolf, N. J. 1973, IAU Symposium No. 52, X-and Gamma-Ray A stronomy, ed. H. Bradt and R. Giacconi (Dordrecht: Reidel), p. 485.

Wynn-Williams, C. G., and Becklin, E. E. 1974, Pub. A.S.P., 86,5 . 